\title{
A CRITERION FOR INTEGRAL DEPENDENCE OF MODULES
}

\author{
BERND ULRICH AND JAVID VALIDASHTI
}

\begin{abstract}
Let $R$ be a universally catenary locally equidimensional Noetherian ring. We give a multiplicity based criterion for an arbitrary finitely generated $R$-module to be integral over a submodule. Our proof is self-contained and implies the previously known numerical criteria for integral dependence of ideals and modules.
\end{abstract}

\section{Introduction}

Let $R$ be a Noetherian ring and $U \subset E$ finitely generated $R$-modules. Our goal is to give a multiplicity based criterion for $U$ to be a reduction of $E$. First assume $U \subset E$ to be submodules of a free $R$-module $F:=R^{e}$. Recall that $U$ is a reduction of $E$ or, equivalently, $E$ is integral over $U$ if $E^{i+1}=U E^{i}$ for some $i \geq 0$, where the products and powers are taken in the polynomial ring $A:=\operatorname{Sym}(F)=R\left[x_{1}, \ldots, x_{e}\right]$. This condition can also be expressed in terms of ring extensions - it means that the inclusion of $R$-subalgebras $R[U] \subset R[E]$ of $A$ is an integral extension. One sometimes calls $R[E]$ the Rees algebra of $E$ (more correctly, of $E \subset F$ ), and when $(R, \mathfrak{m})$ is local we write $\ell(E):=\operatorname{dim} R[E] \otimes_{R} R / \mathfrak{m}$ for the analytic spread of $E$. The analytic spread satisfies the inequality $\ell(E) \leq d+e-1$ if $d:=\operatorname{dim} R>0$, and it gives the smallest possible number of generators of a reduction of the module $E$, at least when $R / \mathfrak{m}$ is infinite. The notions of reduction and analytic spread of a module $E$ do not depend on the embedding $E \subset F$ into a free module, although the algebra $R[E]$ does [5]. To define integral dependence for arbitrary finitely generated $R$-modules $U \subset E$, one maps a free module $R^{e}$ onto the $R$-dual $E^{*}$ of $E$ and replaces $E$ by its image in $R^{e *}$, thus reverting to the case treated above [5]. Hence we may always assume that $U \subset E$ are submodules of a free module.

Multiplicity based criteria for reductions go back to Rees [18], who treated the case of $\mathfrak{m}$-primary ideals in an equidimensional universally catenary Noetherian local ring $(R, \mathfrak{m})$ - two such ideals $J \subset I$ are integral over each other if and only if they have the same Hilbert-Samuel multiplicity. Conversely, whenever this equivalence holds in a Noetherian local ring $R$, then $R$ has to be equidimensional and universally catenary [16]. Rees' result has been generalized in various directions, most notably to modules of finite colength in a free module and, more generally, to ideals and then modules whose quotients have finite length or, yet more generally, have 'sufficiently high' codimension [2], [3], [8], [10], [11], [12], [17], [19], [21] ([8] works for arbitrary modules $U \subset E$, but the codimension conditions are built into the definition of the multiplicity which uses a codimension filtration descending to the integral closure of the module). These criteria are all based on the Hilbert-Samuel or Buchsbaum-Rim

Received by the editors September 14, 2006.

The first author was supported in part by the NSF. 
multiplicity, and variations thereof. The first algebraic criterion that does not rely on any kind of finiteness or codimension condition is due to Flenner and Manaresi [6], who use the concept of $j$-multiplicity introduced by Achilles and Manaresi [1]. However, their result is restricted to the case of ideals and their arguments cannot be extended to modules. The purpose of the present article is to fill this gap and at the same time provide a self-contained, fairly short proof that implies the earlier algebraic results. In the complex-analytic setting Gaffney and Gassler [9] had given a criterion for integral dependence of arbitrary ideals that precedes [6] and uses the Segre numbers defined in [12].

Thus let $(R, \mathfrak{m})$ be a Noetherian local ring of dimension $d$ and $E$ a submodule of a free $R$-module $F:=R^{e}$. We introduce a multiplicity $j(E)$ for $E$ that generalizes the Buchsbaum-Rim multiplicity defined when $E \subset F$ has finite colength as well as the $j$-multiplicity of Achilles-Manaresi that applies when $E \subset R$ is an ideal. We prove that the function

$$
\Sigma(n):=\sum_{i=0}^{n-1} \lambda_{R}\left(\Gamma_{\mathfrak{m}}\left(\frac{E^{i} F^{n-i}}{E^{i+1} F^{n-i-1}}\right)\right)
$$

is polynomial of degree at most $d+e-1$ for $n \gg 0$,

$$
\Sigma(n)=\frac{j(E)}{(d+e-1) !} n^{d+e-1}+\text { lower terms } .
$$

Here $\lambda_{R}(-)$ denotes length and $\Gamma_{\mathfrak{m}}(-)=\mathrm{H}_{\mathfrak{m}}^{0}(-)$ zeroth local cohomology. The multiplicity $j(E)$ so defined is a non-negative integer. It can also be described as

$$
j(E)=(d+e-1) ! \lim _{n \rightarrow \infty} \frac{\Sigma(n)}{n^{d+e-1}} .
$$

If $E \subset F$ has finite colength, then $\Sigma(n)=\lambda_{R}\left(F^{n} / E^{n}\right)$, and hence $j(E)$ is the Buchsbaum-Rim multiplicity. If on the other hand $E \subset R$ is an ideal, then $\Sigma(n)$ is the so-called first sum transform of the function $\lambda_{R}\left(\Gamma_{\mathfrak{m}}\left(E^{n} / E^{n+1}\right)\right)$ used in the definition of the $j$-multiplicity of an ideal and thus again our multiplicity coincides with the earlier one. One may be tempted to define a multiplicity $j(E)$ based on the function $n \mapsto \lambda_{R}\left(\Gamma_{\mathfrak{m}}\left(F^{n} / E^{n}\right)\right)$ instead. This approach however fails, as the simpler function need not be polynomial eventually [4].

Our main result is that the new multiplicity can indeed be used to detect integral dependence of modules:

Theorem 1.1. Let $R$ be a universally catenary locally equidimensional Noetherian ring, let $U \subset E$ be submodules of a free $R$-module $F:=R^{e}$, and assume that $U_{\mathfrak{p}}=F_{\mathfrak{p}}$ for every minimal prime $\mathfrak{p}$ of $R$. The following are equivalent:

(i) $j\left(U_{\mathfrak{q}}\right)=j\left(E_{\mathfrak{q}}\right)$ for every $\mathfrak{q} \in \operatorname{Spec}(R)$.

(ii) $j\left(U_{\mathfrak{q}}\right) \leq j\left(E_{\mathfrak{q}}\right)$ for every $\mathfrak{q} \in \operatorname{Supp}_{R}(F / U)$ with $\operatorname{dim} R_{\mathfrak{q}}=\ell\left(U_{\mathfrak{q}}\right)-e+1$.

(iii) $U$ is a reduction of $E$.

One easily sees that the set of primes considered in (ii) is finite (Remark 4.2).

The case where $U \subset E$ are ideals is the afore mentioned criterion of Flenner and Manaresi [6, 3.3]. However, our proof is different and recovers the earlier result for ideals without the use of hyperplane sections. We deduce Theorem 1.1 from a more general theorem - a multiplicity based criterion for integral dependence of ideals generated by linear forms in a graded $R$-algebra $A$ (Theorem 3.4). The difference to the 
Flenner-Manaresi result is that our criterion for such ideals is local in the primes of the ground ring $R$, whereas theirs involves primes of the algebra $A$ and would not suffice to prove Theorem 1.1 (Remark 4.6). We make use of two multiplicities associated to ideals generated by linear forms, which we call $j^{*}$ and $j^{\#}$. They coincide in most cases (Proposition 3.2), but serve different purposes. The first multiplicity is more readily seen to be additive on short exact sequences of graded modules (Theorem 2.5), and this immediately implies that it remains constant when passing to a reduction (Theorem 2.6). The second multiplicity on the other hand is more suited for proving that, conversely, the constancy of the multiplicity implies integral dependence (Theorem $3.3(\mathrm{~b})$ ); furthermore it contains as a special case the $j$-multiplicity of a module, which in turn generalizes the Buchsbaum-Rim as well as the Achilles-Manaresi multiplicity. The main idea behind the definition of $j^{*}$ and $j^{\#}$ is to consider an 'internal' grading on associated graded rings and modules that is induced by the grading of the ambient ring $A$. Our approach is partly inspired by [21].

\section{The multiplicity $j^{*}$}

We begin by recalling the notion of $j$-multiplicity for graded modules as introduced and developed in $[7,6.1]$. Let $R$ be a Noetherian ring with a fixed maximal ideal $\mathfrak{m}$, and $S$ a standard graded Noetherian $R$-algebra, i.e., a graded $R$-algebra with $S_{0}=R$ that is generated by finitely many homogeneous elements of degree one. Consider a finitely generated graded $S$-module $N$. Notice that $\Gamma_{\mathfrak{m}}(N)$ is a graded $S$-submodule of $N$. In particular, $\Gamma_{\mathfrak{m}}(N)$ is finitely generated over $S$. Thus there exists a fixed power $\mathfrak{m}^{s}$ of $\mathfrak{m}$ that annihilates it, and then $\Gamma_{\mathfrak{m}}(N)$ can be regarded as a finitely generated graded module over $S / \mathfrak{m}^{s} S$, a standard graded Noetherian algebra over the Artinian local ring $R / \mathfrak{m}^{s}$. Hence $\Gamma_{\mathfrak{m}}(N)$ has a Hilbert function that is eventually polynomial of degree at most $\operatorname{dim} N-1$ and gives the multiplicity $e\left(\Gamma_{\mathfrak{m}}(N)\right)$. Now let $D$ be any integer with $D \geq \operatorname{dim} N$. One defines the $j$-multiplicity $j_{D}(N)$ of the graded module $N$ to be $e\left(\Gamma_{\mathfrak{m}}(N)\right)$ when $D=\operatorname{dim} \Gamma_{\mathfrak{m}}(N)$ and zero otherwise. It easily follows that $j_{D}(N)=0$ if (and only if) $\operatorname{dim} N / \mathfrak{m} N<D$, a fact we will use frequently in this paper. Also notice that

$$
j_{D}(N)=(D-1) ! \lim _{n \rightarrow \infty} \frac{\lambda_{R}\left(\Gamma_{\mathfrak{m}}\left(N_{n}\right)\right)}{n^{D-1}} .
$$

If $D=\operatorname{dim} N$ one simply writes $j(N)$ instead of $j_{D}(N)$.

Remark 2.1. Later we will consider the set of prime ideals $\left\{\mathfrak{q} \in \operatorname{Spec}(R) \mid j\left(N_{\mathfrak{q}}\right) \neq 0\right\}$ where the $j$-multiplicity does not vanish. Notice that this set is finite as each of its elements is contracted from a minimal prime in $\operatorname{Supp}_{S}(N)$.

We will only use the relatively easy fact about $j$-multiplicities that they are additive on short exact sequences of graded modules, see [7, 6.1.2]:

Proposition 2.2. If $0 \rightarrow N^{\prime} \rightarrow N \rightarrow N^{\prime \prime} \rightarrow 0$ is an exact sequence of finitely generated graded $S$-modules and $D$ is an integer with $D \geq \operatorname{dim} N$, then

$$
j_{D}(N)=j_{D}\left(N^{\prime}\right)+j_{D}\left(N^{\prime \prime}\right) .
$$


We are now ready to introduce the multiplicity $j^{*}$. The main idea here is to consider a suitable grading on extended Rees modules that we call 'internal grading'. A similar grading has been used in $[13,8.1 .3]$.

Definition and Discussion 2.3. Let $(R, \mathfrak{m})$ be a Noetherian local ring, $A$ a standard graded Noetherian $R$-algebra, $I$ an $A$-ideal generated by linear forms, and $M$ a finitely generated graded $A$-module.

Let $t$ be a variable. Using the convention that $I^{i}=A$ whenever $i \leq 0$, we write

$$
\mathcal{R}_{I}(M)^{+}:=\oplus_{i \in \mathbb{Z}} M I^{i} t^{i} \subset M \otimes_{R} R\left[t, t^{-1}\right]
$$

for the extended Rees module of $M$ with respect to $I$ or of $I$ on $M$. This is a module over the extended Rees ring $\mathcal{R}_{I}(A)^{+}$. It gives rise to the associated graded module of $M$ with respect to $I$ or of $I$ on $M$,

$$
G_{I}(M):=\mathcal{R}_{I}(M)^{+} / t^{-1} \mathcal{R}_{I}(M)^{+}=\oplus_{i=0}^{\infty} I^{i} M / I^{i+1} M,
$$

which is a module over the associated graded ring $G_{I}(A)$ of the same dimension as $M$.

Assigning degree zero to the variable $t$, the Laurent polynomial ring $A\left[t, t^{-1}\right]$ becomes a standard graded Noetherian $R\left[t, t^{-1}\right]$-algebra, and $M\left[t, t^{-1}\right]:=M \otimes_{R} R\left[t, t^{-1}\right]$ a finitely generated graded module over this algebra. The extended Rees $\operatorname{ring} \mathcal{R}_{I}(A)^{+}$ is a homogeneous $R\left[t^{-1}\right]$-subalgebra of $A\left[t, t^{-1}\right]$, and hence a standard graded Noetherian $R\left[t^{-1}\right]$-algebra. Furthermore $\mathcal{R}_{I}(M)^{+}$is a graded $\mathcal{R}_{I}(A)^{+}$-submodule of $M\left[t, t^{-1}\right]$, thus a finitely generated graded module over $\mathcal{R}_{I}(A)^{+}$. With respect to this grading, $G_{I}(A)=\mathcal{R}_{I}(A)^{+} / t^{-1} \mathcal{R}_{I}(A)^{+}$becomes a standard graded Noetherian $R$-algebra and $G_{I}(M)=\mathcal{R}_{I}(M)^{+} / t^{-1} \mathcal{R}_{I}(M)^{+}$a finitely generated graded module over this algebra. Notice that

$$
\left[G_{I}(M)\right]_{n}=\oplus_{i=0}^{\infty}\left[I^{i} M / I^{i+1} M\right]_{n} .
$$

The grading so defined on the extended Rees module and the associated graded module will be referred to as internal grading - for it is induced by the grading on the module $M$.

Now let $D$ be an integer with $D \geq \operatorname{dim} M$. We define the $j^{*}$-multiplicity of $M$ with respect to $I$ or of $I$ on $M$, as

$$
j_{D}^{*}(I, M):=j_{D}\left(G_{I}(M)\right),
$$

where $G_{I}(M)$ is graded by the internal grading. Again notice that

$$
j_{D}^{*}(I, M)=(D-1) ! \lim _{n \rightarrow \infty} \frac{\sum_{i=0}^{\infty} \lambda_{R}\left(\Gamma_{\mathfrak{m}}\left(\left[I^{i} M / I^{i+1} M\right]_{n}\right)\right)}{n^{D-1}} .
$$

If $D=\operatorname{dim} M$ we simply write $j^{*}(I, M)$ instead of $j_{D}^{*}(I, M)$.

Remark 2.4. In the setting of Definition 2.3 one can also consider the usual $j$ multiplicity $j_{D}(I, M)$ in the sense of $[1,1.2]$ and $[7,6.1 .5]$. It is defined as $j_{D}\left(G_{I}(M) \otimes\right.$ $\left.{ }_{A} A_{\mathfrak{n}}\right)$, where $\mathfrak{n}$ denotes the homogeneous maximal ideal of $A$ and $G_{I}(M)$ is endowed with the usual grading, assigning degree zero to the elements of $A$ and degree one to t. Again one writes $j(I, M):=j_{D}(I, M)$ when $D=\operatorname{dim} M$. This notion does not coincide with ours and, as we will see in Remark 4.6, would not be suitable for the purpose of the present paper. 
For example, let $R=k\left[\left[y_{1}, \ldots, y_{d}\right]\right]$ be a power series ring over a field $k$ in $d>$ 0 variables $y_{1}, \ldots, y_{d}, M:=A:=R\left[x_{1}, \ldots, x_{d}\right]$ a polynomial ring over $R$ in the variables $x_{1}, \ldots, x_{d}$, and $I$ the $A$-ideal $\left(y_{i} x_{i} \mid 1 \leq i \leq d\right)$. With the internal grading $G_{I}(M)$ is again a standard graded algebra over $(R, \mathfrak{m})$. In fact, it is a polynomial ring $(A / I)\left[z_{1}, \ldots, z_{d}\right]$ because $I$ is a complete intersection. The ideal $\Gamma_{\mathfrak{m}}\left(G_{I}(M)\right)$ in this ring is generated by the image, $\bar{x}$ say, of $x_{1} \cdots x_{d}$. Hence it is isomorphic to $G_{I}(A) / \operatorname{ann}_{G_{I}(A)}(\bar{x})$ and so to $k\left[x_{1}, \ldots, x_{d}, z_{1}, \ldots, z_{d}\right]$. Therefore $j^{*}(I, M)=1$. In the usual grading however, the graded components of $G_{I}(M)_{\mathfrak{n}}$ all have positive depth as modules over $\left[G_{I}(A)_{\mathfrak{n}}\right]_{0}=A_{\mathfrak{n}} / I_{\mathfrak{n}}$, hence their zeroth local cohomology vanishes. Thus $j(I, M)=0$.

We will need the fact that the multiplicity $j^{*}$ is additive on short exact sequences:

Theorem 2.5. Let $R$ be a Noetherian local ring, $A$ a standard graded Noetherian $R$-algebra, and $I$ an $A$-ideal generated by linear forms. If $0 \rightarrow M^{\prime} \rightarrow M \rightarrow M^{\prime \prime} \rightarrow 0$ is an exact sequence of finitely generated graded $A$-modules and $D$ is an integer with $D \geq \operatorname{dim} M$, then

$$
j_{D}^{*}(I, M)=j_{D}^{*}\left(I, M^{\prime}\right)+j_{D}^{*}\left(I, M^{\prime \prime}\right) .
$$

Proof. The proof is similar to that of [7, 1.2.6 and 6.1.7]. Throughout we consider all extended Rees modules as graded by the internal grading. Thus $\mathcal{R}_{I}(A)^{+}$is a standard graded $R\left[t^{-1}\right]$-algebra. When computing $j$-multiplicities of graded modules over this algebra we use the maximal ideal $\left(\mathfrak{m}, t^{-1}\right)$ of $R\left[t^{-1}\right]$, where $\mathfrak{m}$ stands for the maximal ideal of $R$. Also notice that $t^{-1}$ is homogeneous of degree zero.

One has the following commutative diagram of graded $\mathcal{R}_{I}(A)^{+}$-modules with exact rows and columns,

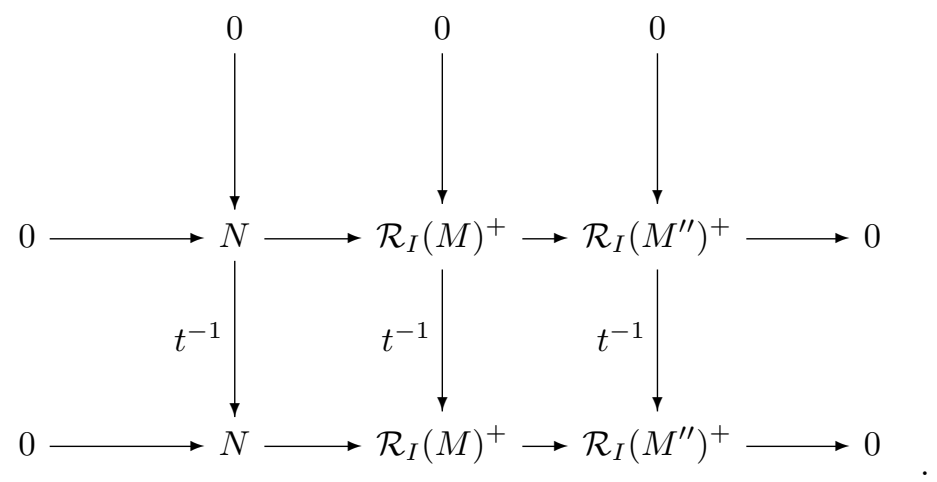

The Snake Lemma yields an exact sequence of finitely generated graded $\mathcal{R}_{I}(A)^{+}{ }_{-}$ modules of dimension at most $D$,

$$
0 \longrightarrow N / t^{-1} N \longrightarrow G_{I}(M) \longrightarrow G_{I}\left(M^{\prime \prime}\right) \longrightarrow 0 \text {. }
$$


Next consider the following commutative diagram with exact rows and columns,

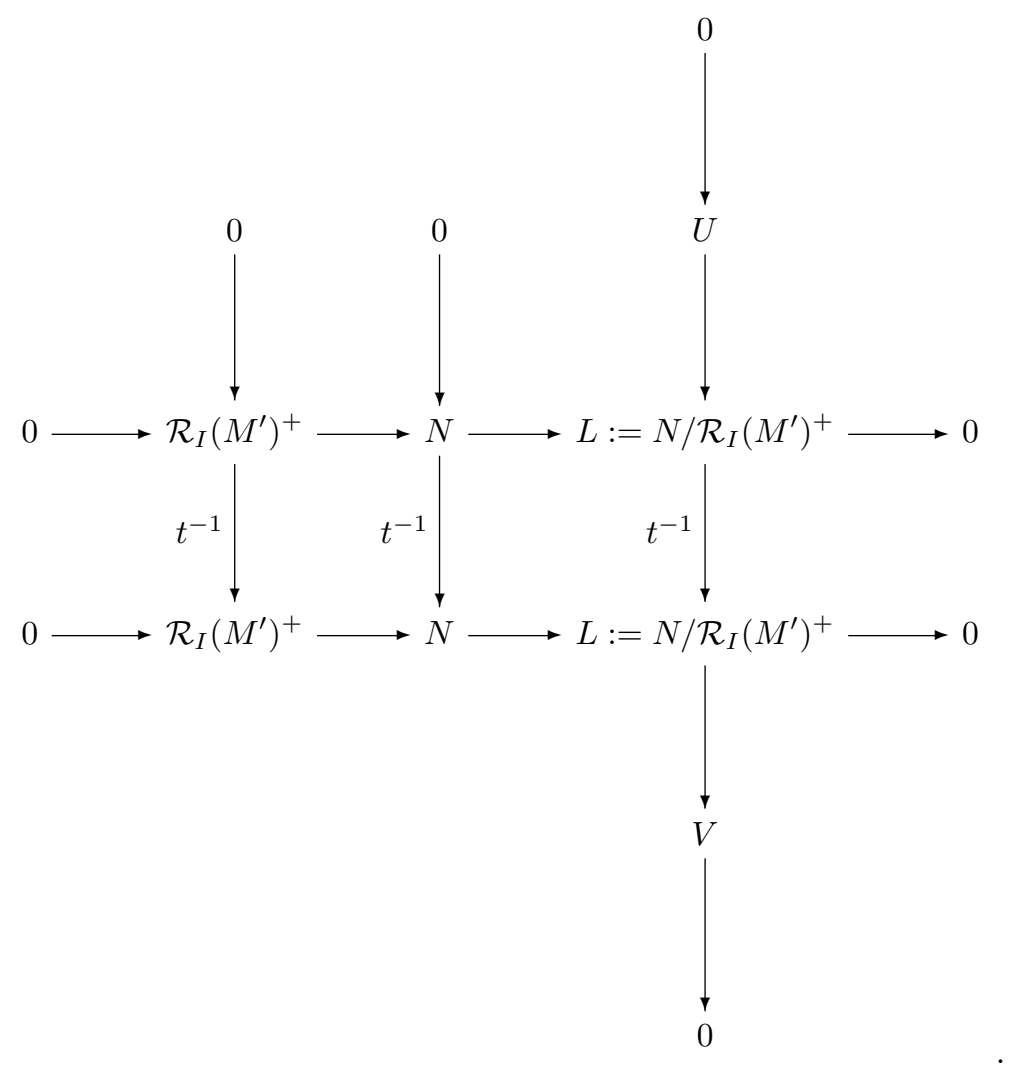

Again from the Snake Lemma we obtain the exact sequence of finitely generated graded $\mathcal{R}_{I}(A)^{+}$-modules having dimension at most $D$,

$$
0 \longrightarrow U \longrightarrow G_{I}\left(M^{\prime}\right) \longrightarrow N / t^{-1} N \longrightarrow V \longrightarrow 0 \text {. }
$$

The above diagram also yields the exact sequence of finitely generated graded $\mathcal{R}_{I}(A)^{+}$modules

$$
0 \longrightarrow U \longrightarrow L \stackrel{t^{-1}}{\longrightarrow} L \longrightarrow V \longrightarrow 0
$$

For $i \leq 0$ the coefficient modules of $t^{i}$ in $\mathcal{R}_{I}\left(M^{\prime}\right)^{+}$and in $N$ coincide, hence the action of $t^{-1}$ on $L$ is nilpotent. Therefore the dimension of $L$ is at most that of $N / t^{-1} N$, which is bounded by $D$. Thus all modules occurring in the exact sequence (3) have dimension at most $D$.

Now (1), (2) and (3) are exact sequences of finitely generated graded $\mathcal{R}_{I}(A)^{+}$modules of dimension at most $D$. Hence we may compute the $j$-multiplicity $j_{D}$ of graded modules along these sequences. Using the additivity of this multiplicity as stated in Proposition 2.2 we deduce that indeed

$$
j_{D}^{*}(I, M)=j_{D}^{*}\left(I, M^{\prime}\right)+j_{D}^{*}\left(I, M^{\prime \prime}\right) .
$$


We will use the previous theorem to prove that the multiplicity $j^{*}$ does not change when passing to a reduction. For $J \subset I$ two $A$-ideals and $M$ a Noetherian $A$-module, we say that $J$ is a reduction of $I$ on $M$ or $I$ is integral over $J$ on $M$ if $I^{i+1} M=J I^{i} M$ for some $i \geq 0$.

Theorem 2.6. Let $A$ be a standard graded Noetherian algebra over a Noetherian local ring, $J \subset I$ A-ideals generated by linear forms, $M$ a finitely generated graded $A$-module, and $D$ an integer with $D \geq \operatorname{dim} M$. If $J$ is a reduction of $I$ on $M$ then $j_{D}^{*}(J, M)=j_{D}^{*}(I, M)$.

Proof. Let $i \geq 0$ be an integer such that $I\left(I^{i} M\right)=I^{i+1} M=J\left(I^{i} M\right)$. One has $G_{I}\left(I^{i} M\right)=G_{J}\left(I^{i} M\right)$ and thus $j_{D}^{*}\left(I, I^{i} M\right)=j_{D}^{*}\left(J, I^{i} M\right)$. On the other hand, set $M_{k}:=I^{k} M / I^{k+1} M$ for $k \geq 0$. Notice that $I M_{k}=0=J M_{k}$, hence $G_{I}\left(M_{k}\right)=M_{k}=$ $G_{J}\left(M_{k}\right)$ and then $j_{D}^{*}\left(I, M_{k}\right)=j_{D}^{*}\left(J, M_{k}\right)$.

Using the additivity of the multiplicity $j^{*}$ as proved in Theorem 2.5, we now conclude that

$$
\begin{aligned}
j_{D}^{*}(I, M) & =j_{D}^{*}\left(I, I^{i} M\right)+\sum_{k=0}^{i-1} j_{D}^{*}\left(I, M_{k}\right) \\
& =j_{D}^{*}\left(J, I^{i} M\right)+\sum_{k=0}^{i-1} j_{D}^{*}\left(J, M_{k}\right) \\
& =j_{D}^{*}(J, M)
\end{aligned}
$$

\section{The multiplicity $j^{\#}$}

To prove a converse of Theorem 2.6 we introduce yet another multiplicity, $j^{\#}$, that is more suited for this purpose. The definition is inspired by [21, 3.2].

Definition and Discussion 3.1. In addition to the assumptions of Definition 2.3 suppose that $M$ is generated in degree zero. Again consider $G_{I}(M)$ as graded by the internal grading. We set

$$
j_{D}^{\#}(I, M):=j_{D}\left(A_{1} G_{I}(M)\right)
$$

and call this integer the $j^{\#-m u l t i p l i c i t y ~ o f ~} M$ with respect to $I$ or of $I$ on $M$.

To be more explicit, the module $A_{1} G_{I}(M)$ has graded components

$$
\left[A_{1} G_{I}(M)\right]_{n}=\oplus_{i=0}^{\infty}\left[I^{i} A_{1} M / I^{i+1} M\right]_{n}=\oplus_{i=0}^{n-1}\left[I^{i} M / I^{i+1} M\right]_{n},
$$

where the last equality holds because $M$ is generated in degree zero. Thus the Hilbert function of $\Gamma_{\mathfrak{m}}\left(A_{1} G_{I}(M)\right)$ is

$$
\Sigma_{I, M}^{\#}(n):=\lambda_{R}\left(\Gamma_{\mathfrak{m}}\left(\left[A_{1} G_{I}(M)\right]_{n}\right)\right)=\sum_{i=0}^{n-1} \lambda_{R}\left(\Gamma_{\mathfrak{m}}\left(\left[I^{i} M / I^{i+1} M\right]_{n}\right)\right) .
$$

The corresponding Hilbert polynomial has degree at most $D-1$ and is of the form

It follows that

$$
\frac{j_{D}^{\#}(I, M)}{(D-1) !} n^{D-1}+\text { lower terms }
$$

$$
j_{D}^{\#}(I, M)=(D-1) ! \lim _{n \rightarrow \infty} \frac{\sum_{i=0}^{n-1} \lambda_{R}\left(\Gamma_{\mathfrak{m}}\left(\left[I^{i} M / I^{i+1} M\right]_{n}\right)\right)}{n^{D-1}} .
$$

Again if $D=\operatorname{dim} M$ we write $j^{\#}(I, M)$ instead of $j_{D}^{\#}(I, M)$. 
Even when $R$ is not local, one has as an immediate consequence of Remark 2.1 that the set $\left\{\mathfrak{q} \in \operatorname{Spec}(R) \mid j^{\#}\left(I_{\mathfrak{q}}, M_{\mathfrak{q}}\right) \neq 0\right\}$ is finite and that each of its elements is contracted from a minimal prime in $\operatorname{Supp}_{G_{I}(A)}\left(A_{1} G_{I}(M)\right)$. Hence for $0 \leq i \leq \operatorname{dim} R$ we may define the 'layered multiplicities'

$$
j^{i \#}(I, M):=\sum_{\mathfrak{q}} j^{\#}\left(I_{\mathfrak{q}}, M_{\mathfrak{q}}\right),
$$

where the sum is taken over all prime ideals $\mathfrak{q}$ of $R$ with $\operatorname{dim}\left(R / \operatorname{ann}_{R}(M)\right)_{\mathfrak{q}}=i$.

It will be useful to clarify the relationship between the two multiplicities $j^{*}$ and $j^{\#}$ :

Proposition 3.2. We use the notation of Definition 3.1. If $\operatorname{dim} M / \mathfrak{m} M<D$ then $j_{D}^{*}(I, M)=j_{D}^{\#}(I, M)$.

Proof. Factoring out the annihilator of $M$ we may assume that $M$ is a faithful $A$ module. Again we consider $G_{I}(M)$ as graded by the internal grading. There is an exact sequence of graded $G_{I}(A)$-modules,

$$
0 \longrightarrow A_{1} G_{I}(M) \longrightarrow G_{I}(M) \longrightarrow G_{I}(M) / A_{1} G_{I}(M) \longrightarrow 0 \text {. }
$$

By the additivity of the $j$-multiplicity, Proposition 2.2, we have

$$
j_{D}\left(G_{I}(M)\right)=j_{D}\left(A_{1} G_{I}(M)\right)+j_{D}\left(G_{I}(M) / A_{1} G_{I}(M)\right) .
$$

Recall that $j_{D}\left(G_{I}(M)\right)=j_{D}^{*}(I, M)$ and $j_{D}\left(A_{1} G_{I}(A)\right)=j_{D}^{\#}(I, M)$.

To obtain $j_{D}\left(G_{I}(M) / A_{1} G_{I}(M)\right)=0$ we show that $G_{I}(M) / A_{1} G_{I}(M)+\mathfrak{m} G_{I}(M)$ has dimension less than $D$, in fact that $G_{I}(A) / A_{1} G_{I}(A)+\mathfrak{m} G_{I}(A)$ has dimension less than $D$. Thus write $B:=G_{I}(A) / A_{1} G_{I}(A)$ and notice that $B=\oplus_{n=0}^{\infty}\left[I^{n}\right]_{n}$. Let $\mathfrak{Q}$ be any minimal prime of $B$. We need to prove that $B / \mathfrak{Q}+\mathfrak{m} B$ has dimension less than $D$. As $B$ can be embedded into $A$ there exists a minimal prime $\mathfrak{P} \in \operatorname{Spec}(A)=\operatorname{Supp}_{A}(M)$ that contracts to $\mathfrak{Q}$. The dimension formula for graded domains $[22,1.1 .2]$ shows that $\operatorname{dim} B / \mathfrak{Q} \leq \operatorname{dim} A / \mathfrak{P}$. Thus if $\operatorname{dim} A / \mathfrak{P}<D$ then indeed $\operatorname{dim} B / \mathfrak{Q}+\mathfrak{m} B<D$. If on the other hand $\operatorname{dim} A / \mathfrak{P}=D$ then $\mathfrak{P}$ cannot contain $\mathfrak{m}$ because $\operatorname{dim} A / \mathfrak{m} A<D$. Thus $\mathfrak{Q}$ does not contain $\mathfrak{m}$ either and we conclude that $\operatorname{dim} B / \mathfrak{Q}+\mathfrak{m} B<\operatorname{dim} B / \mathfrak{Q} \leq$ $\operatorname{dim} A / \mathfrak{P} \leq D$.

Returning to the example of Remark 2.4, the above proposition immediately gives that $j^{\#}(I, M)=j^{*}(I, M)=1$ in this case.

The next theorem provides a crucial step in the proof of our main result.

Theorem 3.3. Let $(R, \mathfrak{m})$ be a Noetherian local ring, A a standard graded Noetherian $R$-algebra, $J \subset I$ A-ideals generated by linear forms, and $M$ a graded $A$ module generated by finitely many homogeneous elements of degree zero. Assume that $\operatorname{dim} M / \mathfrak{m} M<\operatorname{dim} M$ and $J_{\mathfrak{q}}$ is a reduction of $I_{\mathfrak{q}}$ on $M_{\mathfrak{q}}$ for every prime $\mathfrak{q}$ of $R$ with $\mathfrak{q} \neq \mathfrak{m}$.

(a) $j^{\#}(J, M) \geq j^{\#}(I, M)$.

(b) Suppose that $R$ is universally catenary, $M$ is equidimensional as an $A$-module, and $\left(I_{1}\right)_{\mathfrak{p}}=\left(A_{1}\right)_{\mathfrak{p}}$ for every prime $\mathfrak{p}$ of $R$ that is the contraction of a minimal prime in $\operatorname{Supp}_{A}(M)$. If $j^{\#}(J, M) \leq j^{\#}(I, M)$ then $J$ is a reduction of $I$ on $M$. 
Proof. We may factor out the annihilator of $M$ to assume that $M$ is a faithful $A$ module, say of dimension $D$. In particular, $A$ is equidimensional of dimension $D$ in the setting of (b). Theorem 2.6 and Proposition 3.2 show that $j^{\#}(J, M)$ does not change when we replace $J$ by the ideal generated by all linear forms in $I$ that are integral over $J$ on $M$. Thus by our assumption on $J$ and $I$ we may suppose that $[I M / J M]_{1}$ has finite length over $R$.

For any $0 \leq i \leq n-1$ consider the following inclusions,

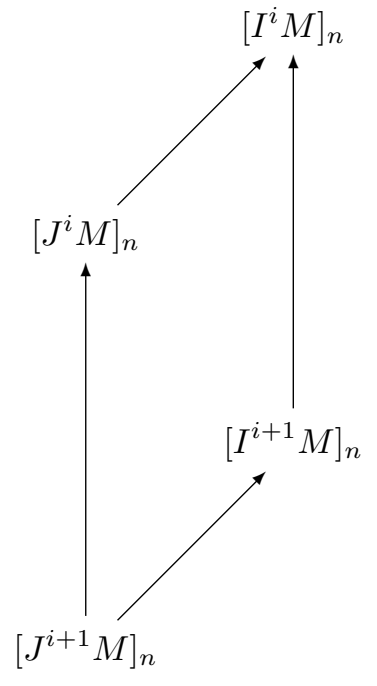

Recall that $[I M / J M]_{1}$ has finite length and that we can dispense of the functor $\Gamma_{\mathfrak{m}}(-)$ for modules of finite length. The left exactness of $\Gamma_{\mathfrak{m}}(-)$ gives

$$
\lambda_{R}\left(\Gamma_{\mathfrak{m}}\left(\left[\frac{J^{i} M}{J^{i+1} M}\right]_{n}\right)\right)+\lambda_{R}\left(\left[\frac{I^{i} M}{J^{i} M}\right]_{n}\right) \geq \lambda_{R}\left(\Gamma_{\mathfrak{m}}\left(\left[\frac{I^{i} M}{J^{i+1} M}\right]_{n}\right)\right) .
$$

On the other hand, since $\Gamma_{\mathfrak{m}}(-)$ is exact on short exact sequences where the left most module has finite length, we obtain

$$
\lambda_{R}\left(\Gamma_{\mathfrak{m}}\left(\left[\frac{I^{i} M}{J^{i+1} M}\right]_{n}\right)\right)=\lambda_{R}\left(\left[\frac{I^{i+1} M}{J^{i+1} M}\right]_{n}\right)+\lambda_{R}\left(\Gamma_{\mathfrak{m}}\left(\left[\frac{I^{i} M}{I^{i+1} M}\right]_{n}\right)\right) .
$$

Taking sums over $i$ in (4) and (5) we deduce that

$$
\Sigma_{J, M}^{\#}(n)+\sum_{i=0}^{n-1} \lambda_{R}\left(\left[\frac{I^{i} M}{J^{i} M}\right]_{n}\right) \geq \sum_{i=1}^{n} \lambda_{R}\left(\left[\frac{I^{i} M}{J^{i} M}\right]_{n}\right)+\Sigma_{I, M}^{\#}(n)
$$

and hence

$$
\Sigma_{J, M}^{\#}(n) \geq \lambda_{R}\left(\left[\frac{I^{n} M}{J^{n} M}\right]_{n}\right)+\Sigma_{I, M}^{\#}(n),
$$

where $\Sigma^{\#}$ is the Hilbert function introduced in Definition 3.1. Recall that this function is eventually polynomial of degree at most $D-1$ and that it gives rise to the $j^{\#}$ multiplicity of a module. 
Comparing coefficients in degree $D-1$ in $(6)$, one sees that $j^{\#}(J, M) \geq j^{\#}(I, M)$, which is part (a). If $j^{\#}(J, M) \leq j^{\#}(I, M)$ then the function $\lambda_{R}\left(\left[I^{n} M / J^{n} M\right]_{n}\right)$ is bounded above by a polynomial of degree at most $D-2$. Part (b) will follow once we have shown that this forces $J$ to be a reduction of $I$ on $M$, provided the assumptions of (b) hold. Here we use the proof of $[21,3.3]$, which we repeat for the convenience of the reader.

Thus consider the standard graded $R$-algebra $B:=R\left[I_{1}\right]=\oplus_{n=0}^{\infty}\left[I^{n}\right]_{n} \subset A$ and the graded $B$-module $N:=B M_{0}=\oplus_{n=0}^{\infty}\left[I^{n} M\right]_{n} \subset M$, which is generated by finitely many homogeneous elements of degree zero. Again we consider the internal grading on the associated graded module $G_{J_{1} B}(N)$. As $[I M / J M]_{1}$ has finite length over $R$, it follows that $B_{1} G_{J_{1} B}(N)=\Gamma_{\mathfrak{m}}\left(B_{1} G_{J_{1} B}(N)\right)$ and that the Hilbert function of this module is $\Sigma_{J_{1} B, N}^{\#}(n)=\lambda_{R}\left(\left[N / J_{1}^{n} N\right]_{n}\right)=\lambda_{R}\left(\left[I^{n} M / J^{n} M\right]_{n}\right)$. If the latter function is bounded by a polynomial of degree at most $D-2$, we conclude that $B_{1} G_{J_{1} B}(N)$ has dimension less than $D$ as a module over $G_{J_{1} B}(B)$.

Next we claim that the $B$-module $N$ is equidimensional of dimension $D$. Indeed, as $M=A N_{0}$ is $A$-faithful and $B \subset A$, it follows that $N$ is $B$-faithful. Hence it suffices to show that $B$ is equidimensional of dimension $D$. Thus let $\mathfrak{Q}$ be a minimal prime of $B$. There exists a minimal prime $\mathfrak{P}$ of $A$ lying over it because $B \subset A$. Write $\mathfrak{p}$ for the contraction of $\mathfrak{P}$ to $R$. Since $\left(I_{1}\right)_{\mathfrak{p}}=\left(A_{1}\right)_{\mathfrak{p}}$ by our assumption, we obtain $(B / \mathfrak{Q})_{\mathfrak{p}}=(A / \mathfrak{P})_{\mathfrak{p}}$. Hence the dimension formula for graded domains $[22,1.1 .2]$ gives

$$
\operatorname{dim} B / \mathfrak{Q}=\operatorname{dim} R / \mathfrak{p}+\operatorname{trdeg}_{R / \mathfrak{p}} B / \mathfrak{Q}=\operatorname{dim} R / \mathfrak{p}+\operatorname{trdeg}_{R / \mathfrak{p}} A / \mathfrak{P}=\operatorname{dim} A / \mathfrak{P} .
$$

But the latter dimension is $D$, showing that the $B$-module $N$ is indeed equidimensional of dimension $D$. Since $R$ is universally catenary, the $G_{J_{1} B}(B)$-module $G_{J_{1} B}(N)$ is then equidimensional of dimension $D$ as well - a standard fact proved by passing through the extended Rees module [16, proof of 3.8].

Finally, as $B_{1} G_{J_{1} B}(N)$ has dimension less than $D$ and $G_{J_{1} B}(N)$ is equidimensional of dimension $D$, we conclude that $B_{1} G_{J_{1} B}(B)$ is contained in every minimal prime of the annihilator of $G_{J_{1} B}(N)$. Hence a power of $B_{1} G_{J_{1} B}(B)$ annihilates $G_{J_{1} B}(N)$. In particular, a power of $B_{1} B / J_{1} B=I_{1} B / J_{1} B$ annihilates $N / J_{1} N$, showing that $J$ is a reduction of $I$ on $M$.

We are now ready to assemble the proof of our main theorem.

Theorem 3.4. Let $R$ be a universally catenary Noetherian ring, A a standard graded Noetherian $R$-algebra, $J \subset I$ A-ideals generated by linear forms, and $M$ a graded A-module generated by finitely many homogeneous elements of degree zero. Assume that the A-module $M$ is equidimensional locally at every maximal ideal of $R$, and $\left(J_{1}\right)_{\mathfrak{p}}=\left(A_{1}\right)_{\mathfrak{p}}$ for every prime $\mathfrak{p}$ of $R$ that is the contraction of a minimal prime in $\operatorname{Supp}_{A}(M)$. The following are equivalent :

(i) $j^{\#}\left(J_{\mathfrak{q}}, M_{\mathfrak{q}}\right)=j^{\#}\left(I_{\mathfrak{q}}, M_{\mathfrak{q}}\right)$ for every prime ideal $\mathfrak{q}$ of $R$.

(ii) $j^{\#}\left(J_{\mathfrak{q}}, M_{\mathfrak{q}}\right) \leq j^{\#}\left(I_{\mathfrak{q}}, M_{\mathfrak{q}}\right)$ for every prime ideal $\mathfrak{q}$ of $R$.

(iii) $j^{i \#}(J, M)=j^{i \#}(I, M)$ for $1 \leq i \leq \operatorname{dim} R$.

(iv) $j^{i \#}(J, M) \leq j^{i \#}(I, M)$ for $1 \leq i \leq \operatorname{dim} R$.

(v) $J$ is a reduction of $I$ on $M$.

Proof. To prove that (v) implies (i) we replace $R$ by any localization $R_{\mathfrak{q}}$ and show that if (v) holds then $j^{\#}(J, M)=j^{\#}(I, M)$. Now $(R, \mathfrak{m})$ is local. If $\operatorname{dim} M / \mathfrak{m} M=\operatorname{dim} M$ 
and $M \neq 0$, then $J_{1}=A_{1}$ by our assumption and hence $J=I$. Thus we may assume that $\operatorname{dim} M / \mathfrak{m} M<\operatorname{dim} M$ as otherwise $M=0$ or $J=I$. Now the asserted implication follows from Theorem 2.6 and Proposition 3.2.

To show that (v) follows from (iv) we let $\mathfrak{q}$ be any prime ideal of $R$. Assuming that (iv) holds we prove by induction on $d:=\operatorname{dim} R_{\mathfrak{q}}$ that $J_{\mathfrak{q}}$ is a reduction of $I_{\mathfrak{q}}$ on $M_{\mathfrak{q}}$. We may suppose $d>0$. Consider the union of $\mathfrak{q}$ and the finitely many primes in $R$ of height $\leq d$ where $j^{\#}(J, M)$ or $j^{\#}(I, M)$ does not vanish. Localizing $R$ at the complement of this union does not change $j^{i \#}(J, M)$ or $j^{i \#}(I, M)$ for $1 \leq i \leq d$. The equidimensionality assumption on $M$ is preserved as well, according to the dimension formula for graded domains $[22,1.1 .2]$. Thus by our induction hypothesis, $J$ is a reduction of $I$ on $M$ locally at every prime in $R$, except for the primes $\mathfrak{m}$ of height $d$. For every such $\mathfrak{m}$ either $M_{\mathfrak{m}}=0$ or $J_{\mathfrak{m}}=I_{\mathfrak{m}}$ or $\operatorname{dim} M_{\mathfrak{m}} / \mathfrak{m} M_{\mathfrak{m}}<\operatorname{dim} M_{\mathfrak{m}}$. In any case $j^{\#}\left(J_{\mathfrak{m}}, M_{\mathfrak{m}}\right) \geq j^{\#}\left(I_{\mathfrak{m}}, M_{\mathfrak{m}}\right)$ according to Theorem 3.3(a). Since this holds for every $\mathfrak{m}$ and since $j^{d \#}(J, M) \leq j^{d \#}(I, M)$ according to (iv), we conclude that $j^{\#}\left(J_{\mathfrak{m}}, M_{\mathfrak{m}}\right)=j^{\#}\left(I_{\mathfrak{m}}, M_{\mathfrak{m}}\right)$ for every prime $\mathfrak{m}$ in $R$ of height $d$. Now Theorem 3.3(b) shows that indeed $J_{\mathfrak{m}}$ is a reduction of $I_{\mathfrak{m}}$ on $M_{\mathfrak{m}}$.

It suffices to verify condition (ii) of Theorem 3.4 for a finite set of prime ideals $\mathfrak{q}$ in $R$ that contains the support of the multiplicity $j^{\#}(J, M)-$ such a set has been identified in Discussion 3.1.

\section{The $j$-multiplicity of a module}

We are now ready to introduce the main object of this paper, the $j$-multiplicity of a module. Here the ideal $I$ of the previous section will be replaced by a module $E$.

Definition and Discussion 4.1. Let $(R, \mathfrak{m})$ be a Noetherian local ring, $E$ a submodule of a free $R$-module $F:=R^{e}$, and $N$ a finitely generated $R$-module of dimension $d$. Write $A:=\operatorname{Sym}(F)$ for the symmetric or Rees algebra of $F$, and consider the $A$-ideal $I:=E A$ and the $A$-module $M:=A \otimes_{R} N$. Notice that $A$ is a polynomial $\operatorname{ring}, I$ is an $A$-ideal generated by linear forms, and $M$ is a finitely generated graded $A$-module of dimension $d+e$ that is generated in degree zero. Thus we may define

$$
j(E, N):=j^{\#}(I, M)
$$

to be the $j$-multiplicity of $E$ on $N$.

To be more explicit,

$$
\left[\frac{I^{i} M}{I^{i+1} M}\right]_{n}=\frac{E^{i} F^{n-i} N}{E^{i+1} F^{n-i-1} N}
$$

for $0 \leq i \leq n-1$, where the products are taken in the $A$-module $M$. Thus the Hilbert function of $\Gamma_{\mathfrak{m}}\left(A_{1} G_{I}(M)\right)$ is

$$
\Sigma(n):=\Sigma_{I, M}^{\#}(n)=\sum_{i=0}^{n-1} \lambda_{R}\left(\Gamma_{\mathfrak{m}}\left(\frac{E^{i} F^{n-i} N}{E^{i+1} F^{n-i-1} N}\right)\right) .
$$

The corresponding Hilbert polynomial has degree at most $d+e-1$ and is of the form

$$
\frac{j(E, N)}{(d+e-1) !} n^{d+e-1}+\text { lower terms . }
$$


It follows that

$$
j(E, N)=(d+e-1) ! \lim _{n \rightarrow \infty} \frac{\sum_{i=0}^{n-1} \lambda_{R}\left(\Gamma_{\mathfrak{m}}\left(E^{i} F^{n-i} N / E^{i+1} F^{n-i-1} N\right)\right)}{n^{d+e-1}} .
$$

If $N=R$ we simply write $j(E)$ instead of $j(E, N)$ - this is the multiplicity described in the introduction.

Finally, for $R$ not necessarily local and $0 \leq i \leq \operatorname{dim} N$, we define

$$
j^{i}(E, N):=j^{i \#}(I, M)=\sum_{\mathfrak{q}} j\left(E_{\mathfrak{q}}, N_{\mathfrak{q}}\right)
$$

the sum being taken over all $\mathfrak{q} \in \operatorname{Supp}_{R}(F N / E N)$ with $\operatorname{dim} N_{\mathfrak{q}}=i$.

As mentioned before, if $E$ has finite colength in $F$ then $j(E)$ is the BuchsbaumRim multiplicity, whereas for $E \subset R$, the case of an ideal, $j(E, N)$ coincides with the $j$-multiplicity $j(E, N)$ as introduced in $[1,1.2]$ and $[7,6.1 .5]$. Notice that like the earlier multiplicities, the $j$-multiplicity $j(E, N)$ of a module $E$ depends on the embedding $E \subset F$ into a free module.

Remark 4.2. We use the setting of Definition 4.1, but without requiring $R$ to be local. In Discussion 3.1 we have seen that the set $\mathcal{A}:=\left\{\mathfrak{q} \in \operatorname{Spec}(R) \mid j\left(E_{\mathfrak{q}}, N_{\mathfrak{q}}\right) \neq 0\right\}$ is finite. If $E_{\mathfrak{p}}=F_{\mathfrak{p}}$ for every minimal prime $\mathfrak{p}$ of $R$, there is another finite set of prime ideals associated to $E$, the locus $\mathcal{B}:=\left\{\mathfrak{q} \in \operatorname{Supp}_{R}(F / E) \mid \ell\left(E_{\mathfrak{q}}\right)=\operatorname{dim} R_{\mathfrak{q}}+e-1\right\}$ where $E$ has 'maximal' analytic spread. To see that this set is finite, choose an element $a \in \operatorname{ann}_{R}(F / E)$ not contained in any minimal prime of $R$ and let $\mathfrak{q} \in \mathcal{B}$. Observe that ht $\mathfrak{q} R_{\mathfrak{q}}\left[E_{\mathfrak{q}}\right] \leq \operatorname{dim} R_{\mathfrak{q}}\left[E_{\mathfrak{q}}\right]-\ell\left(E_{\mathfrak{q}}\right)=1$. Thus the ideals $a R_{\mathfrak{q}}\left[E_{\mathfrak{q}}\right] \subset \mathfrak{q} R_{\mathfrak{q}}\left[E_{\mathfrak{q}}\right]$ both have height one, showing that $\mathfrak{q}$ is contracted from one of the finitely many minimal primes of $a R[E]$.

If $\operatorname{Supp}_{R}(N)=\operatorname{Spec}(R)$ and $E \subset R$ is an ideal, then the two sets $\mathcal{A}$ and $\mathcal{B}$ coincide $[7,6.1 .6(1)]$. However, this is no longer true for modules of higher rank, as can be seen from the example of Remark 2.4, taking $E:=I_{1} \subset F:=A_{1}, N:=R$, and $d=\operatorname{dim} R \geq 2$. Indeed there we have seen that $j(E)=j^{\#}(I, A)=j^{*}(I, A)=1 \neq 0$, whereas $\ell(\bar{E})=e<d+e-1$.

Now let $U$ be an $R$-submodule of $E$ and write $J:=U A$ for the corresponding ideal of $A$. We say that $U$ is a reduction of $E$ on $N$ if $E^{i+1} N=U E^{i} N$ for some $i \geq 0$. This is equivalent to $J$ being a reduction of $I$ on $M$. Thus Theorem 3.4 immediately gives the following version of Theorem 1.1:

Theorem 4.3. Let $R$ be a universally catenary Noetherian ring, $U \subset E$ submodules of a free $R$-module $F:=R^{e}$, and $N$ a finitely generated locally equidimensional $R$ module. Assume that $U_{\mathfrak{p}}=F_{\mathfrak{p}}$ for every minimal prime $\mathfrak{p}$ in $\operatorname{Supp}_{R}(N)$. The following are equivalent :

(i) $j\left(U_{\mathfrak{q}}, N_{\mathfrak{q}}\right)=j\left(E_{\mathfrak{q}}, N_{\mathfrak{q}}\right)$ for every prime ideal $\mathfrak{q}$ of $R$.

(ii) $j\left(U_{\mathfrak{q}}, N_{\mathfrak{q}}\right) \leq j\left(E_{\mathfrak{q}}, N_{\mathfrak{q}}\right)$ for every prime ideal $\mathfrak{q}$ of $R$.

(iii) $j^{i}(U, N)=j^{i}(E, N)$ for $1 \leq i \leq \operatorname{dim} N$.

(iv) $j^{i}(U, N) \leq j^{i}(E, N)$ for $1 \leq i \leq \operatorname{dim} N$.

(v) $U$ is a reduction of $E$ on $N$. 
Remark 4.4. Contrary to what Remark 4.2 might suggest, we may restrict ourselves to the finite set of primes $\mathcal{B}$ for the module $U$ when verifying condition (ii) in Theorem 4.3. For simplicity assume that $\operatorname{Supp}_{R}(N)=\operatorname{Spec}(R)$, a case one can always reduce to by factoring out the annihilator of $N$. Now indeed, as we shall argue, it suffices to require the inequality of Theorem 4.3(ii) for the finitely many prime ideals $\mathfrak{q} \in$ $\operatorname{Supp}_{R}(F / U)$ satisfying $\operatorname{dim} R_{\mathfrak{q}}=\ell\left(U_{\mathfrak{q}}\right)-e+1$.

To see that this condition already implies (v) we may assume $(R, \mathfrak{m})$ is local, $U \neq F$, and $N$ is faithful. We prove by induction on $d:=\operatorname{dim} R$ that $U$ is a reduction of $E$. By our induction hypothesis, $U$ is a reduction of $E$ locally on the punctured spectrum of $R$. If $d=\ell(U)-e+1$ then our assumption gives that condition (ii) in Theorem 4.3 obtains for $\mathfrak{q}=\mathfrak{m}$. As this condition also holds for every $\mathfrak{q} \neq \mathfrak{m}$ by our induction hypothesis and Theorem 4.3, the same theorem implies that $U$ is a reduction of $E$ on $N$. Hence $U$ is a reduction of $E$ because $N$ is faithful. If on the other hand $d \neq \ell(U)-e+1$, one can use $[21,5.6]$ to conclude that again $U$ is a reduction of $E$ (see also [10], [12], [14], [20]).

Remark 4.5. One may be tempted to replace the sequence of multiplicities $j^{i}$ in Theorem 4.3 by the single multiplicity $\sum_{i} j^{i}$ involving all primes of $R$ at the same time, in the spirit of [15, Definition 3]. However, conditions (iii) and (iv) so modified would not suffice anymore to imply integral dependence even when $F / U$ and $F / E$ have the same support.

For instance, let $R:=k[[y, z]]$ be a power series ring over a field, $a, b, c$ positive integers satisfying $b+c \leq a \leq b+b c+c^{2}$, and $U:=\left(y^{a}\right) \subset E:=y^{b}(y, z)^{c} \subset$ $F:=R$. The ideals $U$ and $E$ have the same radical, but are not integral over each other. On the other hand, $j^{1}(U)+j^{2}(U)=j^{1}(U)=a$ and one easily computes that $j^{1}(E)+j^{2}(E)=b+b c+c^{2}$.

Remark 4.6. One can also give a criterion for integral dependence of modules that is based on the Flenner-Manaresi result about integral dependence of ideals. However, such a criterion would not be useful as it is not local in the primes of $R$, but rather involves prime ideals of the polynomial ring $\operatorname{Sym}(F)$.

In fact, using the notation of Definition 4.1 and writing $J \subset I$ for the ideals of $A:=\operatorname{Sym}(F)$ generated by $U$ and $E$, respectively, we recall that $U$ is a reduction of $E$ on $N$ if and only if $J$ is a reduction of $I$ on $M$. Now assume that $R$ is equidimensional and universally catenary and that $\operatorname{Supp}_{R}(N)=\operatorname{Spec}(R)$. The criterion of Flenner and Manaresi for ideals, [6,3.3], gives that $J$ is a reduction of $I$ on $M$ if and only if $j\left(J_{\mathfrak{Q}}, M_{\mathfrak{Q}}\right)=j\left(I_{\mathfrak{Q}}, M_{\mathfrak{Q}}\right)$ for every prime ideal $\mathfrak{Q}$ of the ring $A$ containing $J$ with $\operatorname{dim} A_{\mathfrak{Q}}=\ell\left(J_{\mathfrak{Q}}\right)$. However, such primes $\mathfrak{Q}$ are not necessarily of the form $\left(\mathfrak{q}, A_{1}\right) A$ or $\mathfrak{q} A$ for $\mathfrak{q}=\mathfrak{Q} \cap R$; in fact the first possibility never occurs unless $\operatorname{dim} R_{\mathfrak{q}}=0$. Hence the criterion requires considering genuine primes of the polynomial ring $A$.

\section{Acknowledgement}

The authors would like to thank Terry Gaffney, Dan Katz, and Steve Kleiman for helpful discussions about the material of this paper. 


\section{References}

[1] R. Achilles and M. Manaresi, Multiplicity for ideals of maximal analytic spread and intersection theory, J. Math. Kyoto Univ. 33 (1993), 1029-1046.

[2] E. Böger, Eine Verallgemeinerung eines Multiplizitätensatzes von D. Rees, J. Algebra 12 (1969), $207-215$.

[3] _ Einige Bemerkungen zur Theorie der ganz-algebraischen Abhängigkeit von Idealen, Math. Ann. 185 (1970), 303-308.

[4] S. D. Cutkosky, H. T. Hà, H. Srinivasan, and E. Theodorescu, Asymptotic behavior of the length of local cohomology, Canad. J. Math. 57 (2005), 1178-1192.

[5] D. Eisenbud, C. Huneke, and B. Ulrich, What is the Rees algebra of a module?, Proc. Amer. Math. Soc. 131 (2003), 701-708.

[6] H. Flenner and M. Manaresi, A numerical characterization of reduction ideals, Math. Z. 238 (2001), 205-214.

[7] H. Flenner, L. O'Carroll, and W. Vogel, Joins and Intersections, Springer Monographs in Mathematics, Springer-Verlag, Berlin, 1999.

[8] T. Gaffney, Generalized Buchsbaum-Rim multiplicities and a theorem of Rees, Comm. Algebra 31 (2003), 3811-3827.

[9] T. Gaffney and R. Gassler, Segre numbers and hypersurface singularities, J. Algebraic Geom. 8 (1999), 695-736.

[10] D. Katz, Reduction criteria for modules, Comm. Algebra 23 (1995), 4543-4548.

[11] D. Kirby and D. Rees, Multiplicities in graded rings I: The general theory, Contemp. Math. 159 (1994), 209-267.

[12] S. Kleiman and A. Thorup, A geometric theory of the Buchsbaum-Rim multiplicity, J. Algebra 167 (1994), 168-231.

[13] _ Mixed Buchsbaum-Rim multiplicities, Amer. J. Math. 118 (1996), 529-569.

[14] _ Conormal geometry of maximal minors, J. Algebra 230 (2000), 204-221.

[15] T. Pham and W. V. Vasconcelos, Complexity of the normalization of algebras, Math. Z., to appear.

[16] L. J. Ratliff, On quasi-unmixed local domains, the altitude formula, and the chain condition for prime ideals (II), Amer. J. Math. 92 (1970), 99-144.

[17] _ Locally quasi-unmixed Noetherian rings and ideals of the principal class, Pacific J. Math. 52 (1974), 185-205.

[18] D. Rees, a-transforms of local rings and a theorem on multiplicities of ideals, Proc. Camb. Phil. Soc. 57 (1961), 8-17.

[19] _ Amao's theorem and reduction criteria, J. London Math. Soc. 32 (1985), 404-410.

[20] _ Reduction of modules, Math. Proc. Camb. Phil. Soc. 101 (1987), 431-449.

[21] A. Simis, B. Ulrich, and W. V. Vasconcelos, Codimension, multiplicity and integral extensions, Math. Proc. Camb. Phil. Soc. 130 (2001), 237-257.

[22] A. Simis and W. V. Vasconcelos, Krull dimension and integrality of symmetric algebras, Manuscripta Math. 61 (1988), 63-78.

Department of Mathematics, Purdue University, 150 North University Street, West LAFAYETTE, IN 47907-2067

E-mail address: ulrich@math.purdue.edu

E-mail address: jvalidas@math.purdue.edu 